Further analysis stratified patients according to the histology of their testicular cancer, as standard treatment for nonseminomas involves chemotherapy, whereas treatment for seminomas is usually by radiotherapy alone. The SIR for myeloid leukemia was 6.77 after nonseminoma compared with 2.39 after seminoma. When only nonseminoma survivors diagnosed since 1990 were included in the analysis, the SIR for myeloid leukemia increased to 37.9 , possibly owing to the introduction of etoposide during this period. The authors conclude that survivors of testicular cancer have an increased risk of second malignancy and that the treatment regimen used for testicular cancer can impact on this risk.

Original article Richiardi L et al. (2006) Second malignancies among survivors of germ-cell testicular cancer: a pooled analysis between 13 cancer registries. Int J Cancer 120: 623-631

\section{High prevalence of ghost authorship in industry-initiated trials}

In a Danish cohort study, Gøtzsche et al. have investigated the prevalence of 'ghost authorship' in industry-initiated clinical trials. They defined a 'ghost author' as an individual who contributed to statistical analysis or to the writing of the trial protocol or published manuscript without being identified as an author, as a member of a study group or writing committee, or in the acknowledgments section. By examining original full trial protocols and subsequent primary papers, the authors found evidence of ghost authorship in 33 of 44 (75\%) industry-initiated trials that received approval in the period 1994-1995 (data published 1997-2002). This is in contrast to the results of self-reporting surveys on ghost authorship, which have estimated prevalences of $10-15 \%$.

Statisticians were the ghost authors identified most frequently and most easily. Recent guidelines issued by the International Committee of Medical Journal Editors do not recommend that individuals who have performed statistical analyses be listed as authors unless they have contributed to other aspects of the paper, but do recommend that such persons be acknowledged. Gøtzsche et al. contend that the role of statisticians in analysis of trial data should be more clearly stated, both to improve accountability and to provide more information as to the role of industry in these studies.
The investigators conclude that ghost authorship is common in industry-initiated trials and that the practice serves a commercial purpose. They suggest that adherence to existing guidelines would reduce the prevalence of ghost authorship, and that more journals should state the individual contributions made by each named author of a manuscript.

Original article Gøtzsche PC et al. (2007) Ghost authorship in industry-initiated randomised trials. PLoS Med 4: e19

\section{Correlation of blood vessel type and prognosis in patients with CCRCC}

Recent studies have suggested that increased intratumoral microvascular density (MVD) correlates with poor prognosis in various malignancies, such as breast, lung, nasopharyngeal and prostate cancer; however, its association with clear-cell renal cell carcinoma (CCRCC) has been controversial. A new study by Yao et al. has evaluated the relationship between tumor vasculature in CCRCC and patient survival.

The study included specimens from 78 patients who were diagnosed with CCRCC and underwent nephrectomy between 1983 and 2004. The authors identified two types of microvessels: undifferentiated vessels that were $\mathrm{CD} 31^{+} / \mathrm{CD} 34^{-}$with small or absent lumen, thick walls, and no pericyte coverage; and differentiated vessels that were $\mathrm{CD}_{34}{ }^{+}$. Tissue microarrays were prepared from all 78 samples. Higher differentiated MVD was correlated with lower tumor grade and longer patient survival, while higher undifferentiated MVD was correlated with higher pathologic grades and shorter survival. In fact, a high density of undifferentiated microvessels was an independent prognostic indicator of shorter survival. Patients with low undifferentiated and high differentiated MVD had a significantly longer survival time than did patients with high undifferentiated and low differentiated MVD $(P=0.0029)$.

On the basis of these results, a high differentiated MVD seems to indicate a better prognosis. The authors suggest that undifferentiated blood vessels might have potential as future therapeutic targets.

Original article Yao X et al. (2007) Two distinct types of blood vessels in clear cell renal cell carcinoma have contrasting prognostic implications. Clin Cancer Res 13: 161-169 